\title{
Infrared and Millimeter Views of the Helix: the Nearest, Massive, Neutral Remnant of a Circumstellar Envelope
}

\section{P.J. Huggins}

Physics Department, New York University, New York NY 10003, USA

P. Cox

IAS, Université de Paris XI, 91405 Orsay, France

T. Forveille

Observatoire de Grenoble, B.P. 53X, 38041 Grenoble Cedex, France

R. Bachiller

IGN OAN, Apt 1143, 28800 Alcalá de Henares, Spain

K. Young

SAO, 60 Garden Street, Cambridge, MA 02138, USA

\begin{abstract}
.
We present new, infrared and millimeter views of the Helix nebula which illustrate the critical role of remnant, neutral AGB envelopes in the formation and evolution of planetary nebulae. Large scale $\sim 1000^{\prime \prime}$ mapping of the entire nebula in the CO $(J=2-1)$ line with the CSO reveals the global structure of the envelope. The $\mathrm{CO}$ emission forms the familiar ring structure seen in optical images of the Helix, and indicates a massive remnant with $\gtrsim 50 \%$ the mass of the ionized nebula. High resolution $\mathrm{CO}$ mapping with the IRAM $30 \mathrm{~m}$ telescope shows that the whole envelope is fragmented into an intricate array of small clumps, closely related to the cometary globules seen in the central, ionized cavity. 5-17 $\mu \mathrm{m}$ spectroscopy of the Helix with ISOCAM reveals a remarkable near infrared spectrum, dominated by the pure $(v=0-0)$ rotational lines of $\mathrm{H}_{2}$. The $\mathrm{H}_{2}$ lines are excited to a temperature of $\sim 900 \mathrm{~K}$, and likely arise in warm, outer layers of the small clumps seen in CO. Imaging of the $\mathrm{H}_{2}$ emission with ISOCAM over the whole nebula provides a striking portrait of the fragmented neutral envelope. 3-dimensional views of the envelope are also presented, based on $\mathrm{CO}$ mapping and using 3 -dimensional visualization techniques. Point symmetries dominate the toroidal structure, and suggest an origin for the Helix in equatorial massloss on the AGB, shaped by the action of bipolar outflows or jets.
\end{abstract}




\section{Introduction}

This paper is concerned with the final evolutionary stages of the circumstellar envelopes of AGB stars and their relation to the formation of planetary nebulae $(\mathrm{PNe})$. It is well established from survey observations in $\mathrm{CO}$ that neutral remnants of the envelopes persist well into the ionized nebula phase in a significant number of PNe (Huggins et al. 1996). Here we focus on one example, the Helix nebula, NGC 7293. At a distance of $\sim 150 \mathrm{pc}$, the Helix is easily the nearest PN with a massive remnant envelope, and is therefore an important case for detailed study. We present new views of the Helix in molecular emission lines of $\mathrm{CO}$ and $\mathrm{H}_{2}$, which highlight the structure of the envelope and its role in the formation of the nebula.

The general appearance of the Helix is familiar from optical images of the ionized nebula which can be found in many popular astronomy texts. For comparison with views at other wavelengths we include in Fig. 1 (left panel) an $R$-band image, where the dominant line contributions are from $\mathrm{H} \alpha$ and [N II]. The diameter of the main nebula is $\sim 1 \mathrm{pc}$, and because of its proximity, the angular size is extremely large $\sim 1000^{\prime \prime}$. The expansion time scale from the kinematics and the size is $\sim 8000 \mathrm{yr}$. The central star is now well evolved from the AGB, with $T_{*} \sim 120000 \mathrm{~K}$ and $L_{*} \sim 100 L_{\odot}$.

If one compares the Helix nebula with a standard AGB envelope, it has obviously undergone dramatic changes in the several thousand years since the central star left the AGB. For example, the envelope of the AGB star IRC+10216 (which is at about the same distance from Earth as the Helix) is almost completely molecular, and the gas density and molecular emission decrease rapidly away from the central star (see Lucas \& Guélin, this volume). The most extended emission is seen in CO, which can be detected at low levels out to $\sim 200^{\prime \prime}$ (Huggins et al. 1988). In contrast, in the Helix, the center is fully ionized, the bulk of the gas has moved much farther from the central star, and the density peaks in the periphery. Nevertheless, a substantial part of the circumstellar gas in the Helix still remains in neutral form.

\section{Large Scale Structure in CO}

The first new view of the neutral envelope of the Helix comes from a large scale mapping project (Young et al. 1999) carried out with the $10.4 \mathrm{~m}$ telescope of the Caltech Sub-millimeter Observatory (CSO) in the $230 \mathrm{GHz} \mathrm{CO}(J=2-1)$ line. 3425 spectra have been used to map the entire face of the nebula, at a resolution of $31^{\prime \prime}$ (with $15^{\prime \prime}$ sampling). The map of the velocity integrated CO line intensity is shown in Fig. 2 (left panel).

The large scale structure of the envelope seen in $\mathrm{CO}$ shows the double ring or "helix" morphology familiar from optical images (see Fig. 1), and when they are superposed they show some striking similarities. It is evident that the ionized gas abuts the neutral gas, and that the ionized nebula has formed through photo-ionization of the surface of the neutral envelope by the radiation field of the central star. By the same line of reasoning, it is evident that the shape of the neutral envelope is a key to understanding the morphology of the ionized nebula. 

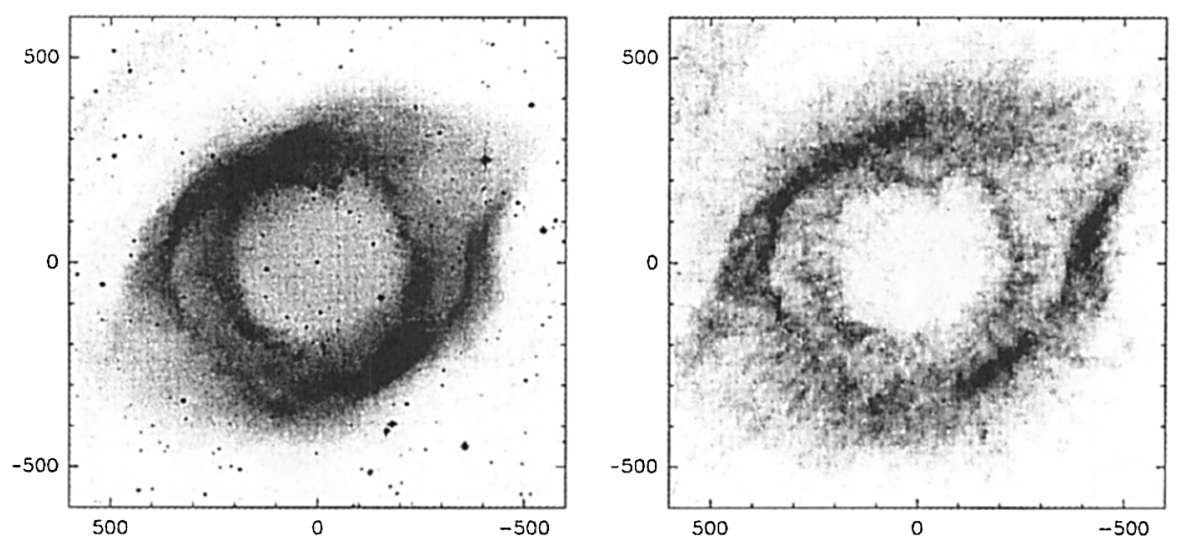

Figure 1. Images of the ionized and neutral components of the Helix nebula. Left panel: $R$-band image of the ionized gas. Right panel: ISOCAM LW2 $(5-8.5 \mu \mathrm{m})$ image, dominated by the pure rotational lines of $\mathrm{H}_{2}$. The offsets are in arcseconds from the center.

The complete CO map is also useful in refining earlier estimates of the mass of the neutral envelope (Huggins \& Healy 1986). The flux can be used to determine the number of $\mathrm{CO}$ molecules in the envelope, and with an appropriate $\mathrm{CO} / \mathrm{H}$ abundance ratio leads to an estimate of the mass. For a $\mathrm{CO} / \mathrm{H}$ ratio in the molecular gas equal to the $\mathrm{C} / \mathrm{H}$ ratio in the ionized gas, the mass of the envelope is $\gtrsim 10 \%$ of the ionized mass. A rough correction for the presence of carbon in other forms can be obtained from the recent detection of $\mathrm{CI}$ emission in the envelope (Young et al. 1997) which increases the mass estimate of the envelope to $\gtrsim 50 \%$ of the ionized mass. Thus the envelope is an important dynamical component of the nebula, as well as a massive reservoir of neutral gas, for much of the evolution of the nebula.

\section{Small Scale Structure in CO}

The second view of the Helix explores the smaller scale structure of the envelope in more detail. Using the IRAM $30 \mathrm{~m}$ telescope we have mapped a $200^{\prime \prime} \times 300^{\prime \prime}$ region of the west limb in the $\mathrm{CO}(J=2-1)$ line, at an angular resolution of $12^{\prime \prime}$ (with $5^{\prime \prime}$ pixels) and a velocity resolution of $0.2 \mathrm{~km} \mathrm{~s}^{-1}$. The linewidth of the whole nebula due to expansion is $\sim 60 \mathrm{~km} \mathrm{~s}^{-1}$.

One example of the $\mathrm{CO}$ velocity channel maps of this region is shown in Fig. 2 (right panel). It can immediately be seen that the gas is highly structured into small clumps, most of which are not resolved by the telescope beam. In addition, in other velocity channel maps of the same area offset by more than 1$2 \mathrm{~km} \mathrm{~s}^{-1}$, the substructure is completely different, indicating very narrow widths to the components. Rough estimates of the masses of the clumps are of order $\sim 10^{-4} M_{\odot}$. Similar clumping is seen in a second field mapped to the North of the central star, so the whole envelope appears to be fragmented into clumps. 

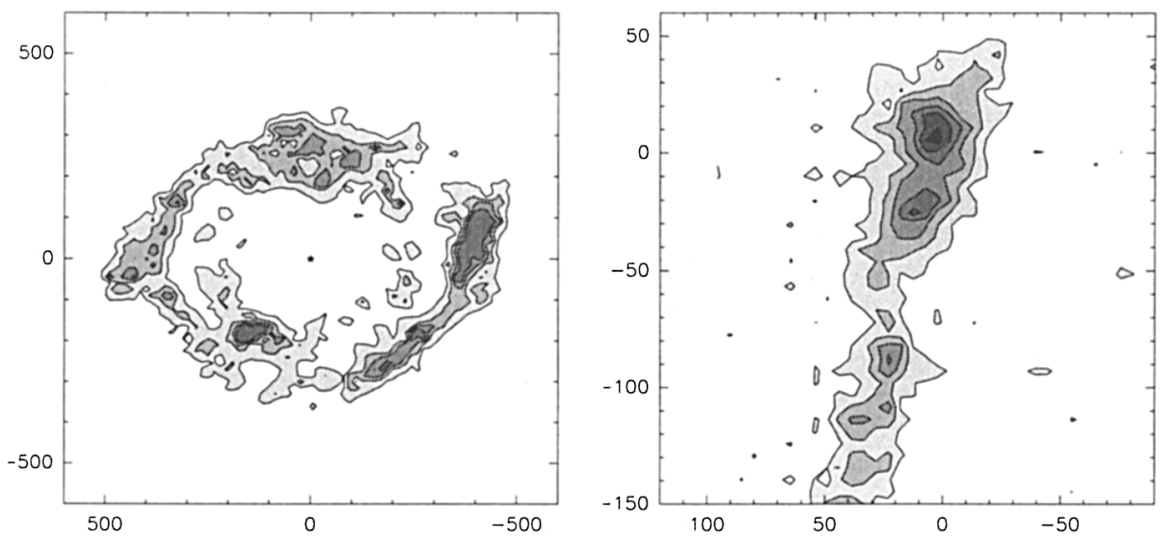

Figure 2. The neutral envelope of the Helix nebula in the $\mathrm{CO}(2-1)$ line. Left panel: Large scale structure in velocity integrated intensity from CSO. Right panel: Small scale structure: channel map $0.2 \mathrm{~km} \mathrm{~s}^{-1}$ wide from the IRAM $30 \mathrm{~m}$ telescope, centered on the west limb.

These structures are obviously close cousins of the well known cometary globules found in the ionized cavity of the Helix (for recent optical images, see Meaburn et al. 1998; O'Dell \& Handron 1996). The fact that we previously found the cometary globules to have cores of molecular gas (Huggins et al. 1992) seals the relationship. Thus the cometary globules require no very special formation scenario, but are rather the innermost remnants of the fragmented neutral envelope.

\section{Molecular Hydrogen}

The third view of the Helix which complements those seen in $\mathrm{CO}$ has been made possible by the ISOCAM instrument on board ISO (Cox et al. 1998). Spectroscopy of the Helix with the CVF $(5-16.6 \mu \mathrm{m})$ reveals that the near infrared spectrum is dominated by the pure rotational $(v=0-0)$ lines of molecular hydrogen. As one moves from the ionized cavity to the neutral envelope, the spectrum changes from one with weak ionized lines, to being dominated by relatively strong lines of $\mathrm{H}_{2}$, from $\mathrm{S}(2)$ to $\mathrm{S}(7)$ (see Cox et al. 1998, Fig. 3). The ortho-para alternation can be seen in the spectra, and from plots of the line intensities vs. excitation levels, the temperature of the gas can be determined. In the small regions studied, the temperature is $\sim 900 \mathrm{~K}$. The $\mathrm{H}_{2}$ is much warmer than the CO, which is typically $\sim 20-50 \mathrm{~K}$ in evolved nebulae (Bachiller et al. 1997), and has a lower column density than the cooler gas, so it is probably present in thin, warm, outer layers around the cool clumps.

The $\mathrm{H}_{2}$ line emission is strong enough to completely dominate the ISOCAM LW2 $(5-8.5 \mu \mathrm{m})$ filter so it has also been possible to image the large scale $\mathrm{H}_{2}$ emission over the whole nebula, with a resolution of $6^{\prime \prime}$. The result is shown in Fig. 1 (right panel). The image confirms the basic picture seen in $\mathrm{CO}$ and the higher resolution shows finer details, including clumps of globules around the 

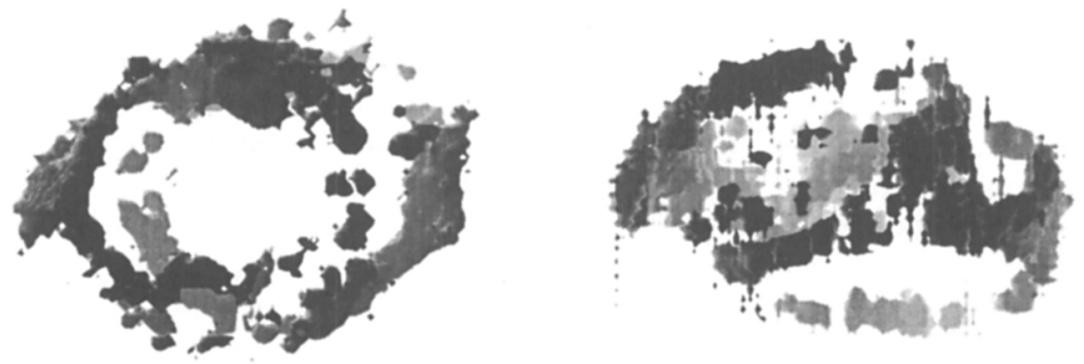

Figure 3. 3-D views of the Helix envelope. A surface contour of the $\mathrm{CO}$ data cube is illuminated diffusely from the rear. Left panel: front view. Right panel: view from below.

inner periphery and flocculent, radial rays extending to large distances. Thus it dramatically underscores the fragmented nature of the shell.

\section{3-D Structure}

The final view of the Helix presented here is a 3-dimensional one. The global geometry is of particular interest for understanding the shaping mechanisms in $\mathrm{PNe}$, and the morphology of the apparent helix has long been a target for model building (e.g., Carranza et al. 1968; Taylor 1977; Huggins \& Healy 1986).

If we return to the large scale CO observations from the CSO, they contain information on the third dimension through the velocities of the $\mathrm{CO}$ components in the spectra. For a homologous radial expansion, expected to dominate in evolved $\mathrm{PNe}$, the observed velocity difference of each component from the systemic velocity is proportional to the displacement from the plane of the sky along the line of sight. Thus the data cube can be used to obtain an approximate 3-dimensional picture of the envelope. In order to visualize the data, a surface contour has been constructed in the 3-dimensional data cube at $10 \%$ of the peak emission, and is illuminated from the rear with diffuse light to show the structure.

The left panel in Fig. 3 shows the front view of the surface. It resembles in outline the conventional map in Fig. 2, but with the illumination the 3dimensional structure begins to become apparent. The darker sections are nearer the observer. An interesting aspect of this approach to visualization is that one can rotate the Helix to view the structure from different directions. Turned to the east or west, the general appearance is that of a distorted torus. The structure is more apparent on turning it through $90^{\circ}$ to view it from below (Fig. 3, right panel). These views show the Helix torus includes a ring, with arcs blown out to each side. The structure also shows a remarkable point symmetry about the 
center: for each feature in the structure, there is a similar one on the opposite side of the central star.

The likely origin of this morphology is mass loss on the AGB towards a preferred equatorial plane, forming the basic toroidal structure, followed by the impact of off-axis, bipolar outflows or jets on the envelope. A striking example of the interaction of bipolar jets with a molecular torus has recently been reported in the young PN KjPn 8 by Forveille et al. (1998), and the Helix may well be a more evolved example of such a system.

\section{Conclusions}

The views of the Helix presented here illustrate the importance of remnant, neutral AGB envelopes in the evolution of PNe. The envelope of the Helix is not only a massive reservoir of neutral gas for the development of the ionized nebula, but is also a key element in both the large and small scale structural features of the nebula. The dominance of the neutral envelope in this and in many similar PNe, also means that the morphology of these nebulae is largely to be understood as the result of processes which shape the neutral envelopes.

Acknowledgments. It is a pleasure to thank the following collaborators on various parts of this work: F. Boulanger, D. Cesarsky, A.P. Jones, B. Lefloch, P.R. Rolfsema, and A.G.G.M. Tielens. This work was supported in part by NSF grant AST96-17941 (to P.J.H.). CSO is funded by the NSF under contract AST96-15025.

\section{References}

Bachiller R., Forveille T., Huggins P.J., Cox P., 1997, A\&A 324, 1123

Carranza G., Courtès G., Louise R., 1968, in Planetary Nebulae, eds. D.E. Osterbrock \& C.R. O'Dell, Reidel, Dordrecht, p. 249

Cox P., Boulanger F., Huggins P.J., Tielens A.G.G.M., Forveille T., Bachiller R., Cesarsky D., Jones A.P., Young K., Roelfsema P.R., Cernicharo J., 1998, ApJ 495, L23

Forveille T., Huggins P.J., Bachiller R., Cox P., 1998, ApJ 495, L111

Huggins P.J., Healy A.P., 1986, ApJ 305, L29

Huggins P.J., Bachiller R., Cox P., Forveille T., 1992, ApJ 401, L43

Huggins P.J., Bachiller R., Cox P., Forveille T., 1996, A\&A 315, 284

Huggins, P.J., Olofsson H., Johansson L.E.B., 1988, ApJ 332, 1009

Meaburn J., Clayton C.A., Bryce M., Walsh J.R., Holloway A.J., Steffen W., 1998, MNRAS 294, 61

O'Dell C.R., Handron K.D., 1996, AJ 111, 1630

Taylor K., 1977, MNRAS 181, 475

Young K., Cox P., Huggins P.J., Forveille T., Bachiller R., 1997, ApJ 482, L101

Young K., Cox P., Huggins P.J., Forveille T., Bachiller R., 1999, in preparation 